\title{
THE BALLOON-BORNE LARGE APERTURE SUBMILLIMETER TELESCOPE (BLAST) 2006: CALIBRATION
} AND FLIGHT PERFORMANCE

\author{
Matthew D. P. Truch ${ }^{1}$, Peter A. R. Ade ${ }^{2}$, James J. Bock ${ }^{3,4}$, Edward L. Chapin ${ }^{5}$, Mark J. Devlin ${ }^{1}$, Simon R. DickeR ${ }^{1}$, \\ Matthew Griffin ${ }^{2}$, Joshua O. Gundersen ${ }^{6}$, Mark Halpern ${ }^{5}$, Peter C. Hargrave ${ }^{2}$, David H. Hughes ${ }^{7}$, Jeff Klein $^{1}$, \\ Gaelen Marsden $^{5}$, Peter G. Martin ${ }^{8,9}$, Philip MauskopF $^{2}$, Lorenzo Moncelsi $^{2}$, C. Barth Netterfield $^{9,10}$, \\ Luca Olmi $^{11,12}$, Enzo Pascale ${ }^{2}$, Guillaume Patanchon ${ }^{13}$, Marie Rex $^{1}$, Douglas ScotT ${ }^{5}$, Christopher Semisch $^{1}$, \\ Nicholas E. Thomas ${ }^{6}$, Carole Tucker ${ }^{2}$, Gregory S. Tucker ${ }^{14}$, Marco P. Viero $^{9}$, and Donald V. Wiebe ${ }^{5,10}$ \\ ${ }^{1}$ Department of Physics and Astronomy, University of Pennsylvania, 209 South 33rd Street, Philadelphia, PA 19104, USA; matthew@ truch.net \\ ${ }^{2}$ Department of Physics \& Astronomy, Cardiff University, 5 The Parade, Cardiff, CF24 3AA, UK \\ 3 Jet Propulsion Laboratory, Pasadena, CA 91109-8099, USA \\ ${ }^{4}$ Observational Cosmology, MS 59-33, California Institute of Technology, Pasadena, CA 91125, USA \\ ${ }^{5}$ Department of Physics \& Astronomy, University of British Columbia, 6224 Agricultural Road, Vancouver, BC V6T 1Z1, Canada \\ ${ }^{6}$ Department of Physics, University of Miami, 1320 Campo Sano Drive, Coral Gables, FL 33146, USA \\ ${ }^{7}$ Instituto Nacional de Astrofísica Óptica y Electrónica (INAOE), Aptdo. Postal 51 y 72000 Puebla, Mexico \\ ${ }^{8}$ Canadian Institute for Theoretical Astrophysics, University of Toronto, 60 St. George Street, Toronto, ON M5S 3H8, Canada \\ ${ }^{9}$ Department of Astronomy \& Astrophysics, University of Toronto, 50 St. George Street, Toronto, ON M5S 3H4, Canada \\ ${ }^{10}$ Department of Physics, University of Toronto, 60 St. George Street, Toronto, ON M5S 1A7, Canada \\ ${ }^{11}$ Physics Department, University of Puerto Rico, Rio Piedras Campus, Box 23343, UPR Station, San Juan, Puerto Rico, USA \\ ${ }^{12}$ INAF-Osservatorio Astrofisico di Arcetri, Largo E. Fermi 5, I-50125 Firenze, Italy \\ ${ }^{13}$ Université Paris Diderot, Laboratoire APC, 10 rue Alice Domon et Léonie Duquet 75205 Paris, France \\ ${ }^{14}$ Department of Physics, Brown University, 182 Hope Street, Providence, RI 02912, USA \\ Received 2009 April 6; accepted 2009 September 30; published 2009 December 7
}

\begin{abstract}
The Balloon-borne Large Aperture Submillimeter Telescope (BLAST) operated successfully during a $250 \mathrm{hr}$ flight over Antarctica in 2006 December (BLAST06). As part of the calibration and pointing procedures, the red hypergiant star VY CMa was observed and used as the primary calibrator. Details of the overall BLAST06 calibration procedure are discussed. The $1 \sigma$ uncertainty on the absolute calibration is accurate to $9.5 \%, 8.7 \%$, and $9.2 \%$ at the 250, 350, and $500 \mu \mathrm{m}$ bands, respectively. The errors are highly correlated between bands resulting in much lower errors for the derived shape of the 250-500 $\mu \mathrm{m}$ continuum. The overall pointing error is $<5^{\prime \prime}$ rms for the $36^{\prime \prime}, 42^{\prime \prime}$, and $60^{\prime \prime}$ beams. The performance of optics and pointing systems is discussed.
\end{abstract}

Key words: balloons - submillimeter - telescopes

Online-only material: color figure

\section{INTRODUCTION}

The 2006 December flight of the Balloon-borne Large Aperture Submillimeter Telescope (BLAST) incorporated a $1.8 \mathrm{~m}$ parabolic primary mirror and large-format bolometer arrays operating at 250,350 , and $500 \mu \mathrm{m}$. A complete description of the BLAST instrument can be found in Pascale et al. (2008). The BLAST bands sample the peak of the spectral energy distribution (SED) for cool dust ( $\sim 10 \mathrm{~K})$. Astronomical signals at these wavelengths are difficult or impossible to access from even the best ground-based sites. As a result, BLAST has the ability to conduct unique Galactic and extragalactic submillimeter surveys with subarcminute resolution and high sensitivity. BLAST's primary scientific motivations are to study the spatial and redshift distribution and evolution of high-redshift star-forming galaxies and to probe the earliest stages of star formation within Galactic molecular clouds.

BLAST conducted a $250 \mathrm{hr}$ flight, launching from McMurdo Station, Antarctica on 2006 December 21, and landing on the Antarctic Plateau on 2007 January 2 (BLAST06). BLAST flew at an average altitude of $38.6 \mathrm{~km}$ with diurnal variations between 37.5 and $39.6 \mathrm{~km}$. Several extragalactic and Galactic fields were mapped, including two large $\left(8.7 \mathrm{deg}^{2}\right)$ and one deep, confusionlimited $\left(0.8 \mathrm{deg}^{2}\right)$ extragalactic fields and two large, overlapping regions (a $50 \mathrm{deg}^{2}$ deep region and a $200 \mathrm{deg}^{2}$ wide region) in the direction of Vela (Devlin et al. 2009; Netterfield et al. 2009).

The primary science goals of the BLAST experiment demand an absolute flux calibration accuracy of $5 \%-10 \%$ in all three BLAST passbands. In particular, a target, uncorrelated 5\% uncertainty, is driven by the extragalactic science case to enable precise measurements of colors and thereby constrain the bolometric luminosities and star formation rates of distant galaxies (Hughes et al. 2002). In order to achieve these goals, a primary calibration source for BLAST with the following properties was required: (1) point-like and bright enough to be detected in each band with a signal-to-noise ratio $(\mathrm{S} / \mathrm{N})$ exceeding $20 \sigma$ in a single map; (2) the absolute (correlated) uncertainty in the SED had to be less than $10 \%$; and (3) the uncorrelated components of the uncertainty in the SED (i.e., uncertainties in the ratios of flux densities in different BLAST bands) could not be greater than $5 \%$. In this paper, we report on the calibration and performance of BLAST06, concentrating on the differences from the calibration procedures used in BLAST05, discussed in Truch et al. (2008, hereafter T08). Section 2 outlines the basic reduction steps and characterization of BLAST06 data. Section 3 discusses the performance of the warm optics in BLAST06. Section 4 outlines the pointing performance of BLAST06. Section 5, the bulk of this paper, describes in detail the absolute calibration (from detector volts to Jy on the sky) derived from the primary flux 


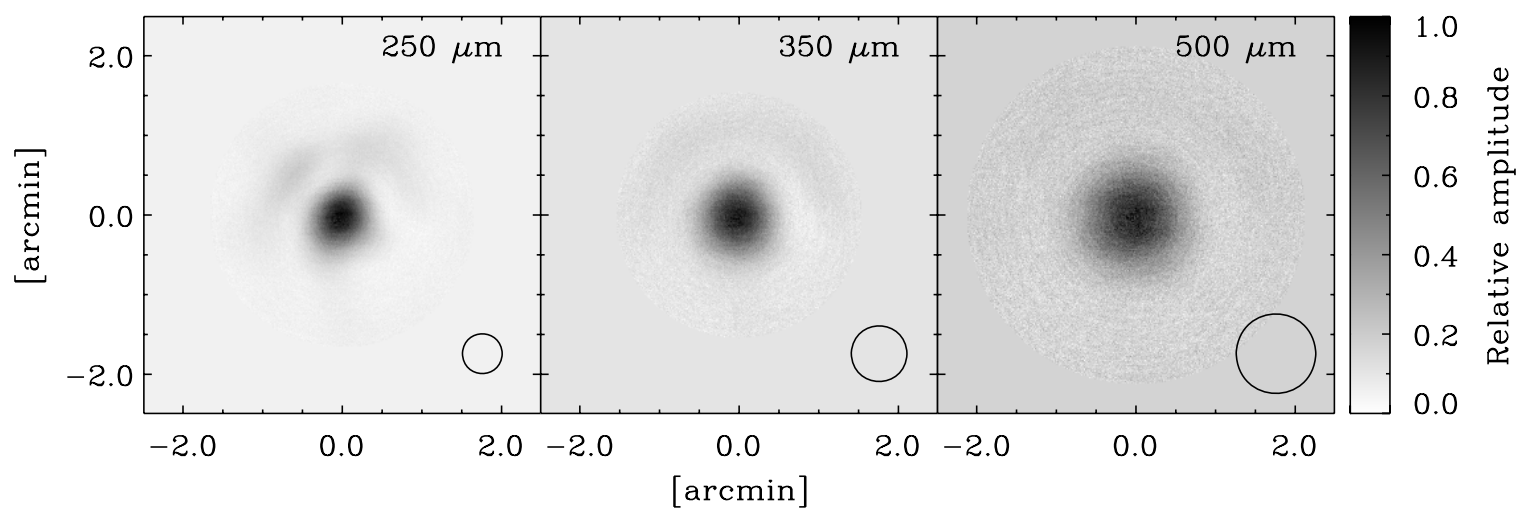

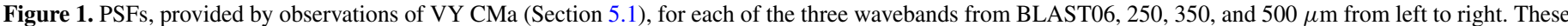

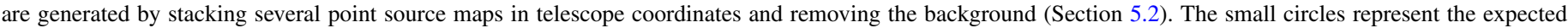

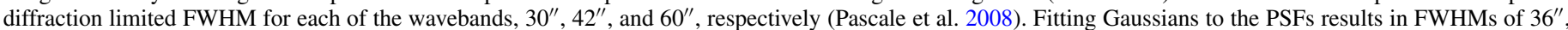

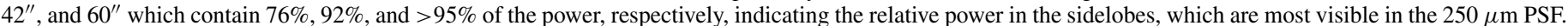

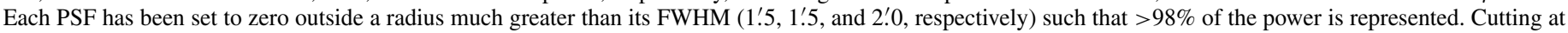
larger radii begins to include residual noise at larger scales not removed by the background template.

calibrator VY CMa (Hoffmeister 1931; Guthnick \& Schneller 1939).

\section{DATA REDUCTION}

The data reduction for BLAST are discussed in detail in Pascale et al. (2008), Patanchon et al. (2008), and T08. Briefly, the data from BLAST consist of a set of 288 bolometer time streams, in voltage units, sampled at $100 \mathrm{~Hz}$. Eighteen of these time streams are diagnostic channels, useful for removing common mode noise, the remaining 270 are coupled to the telescope. These bolometer data are first cleaned for post-flight analysis by being de-spiked and then deconvolved to remove the effects of the data acquisition system filters from the time streams. The cleaned data are then combined with a post-flight pointing solution (Pascale et al. 2008) to make maps at each wavelength. The map-making process takes advantage of the multiple detectors, as well as significant scan cross-linking, to minimize striping due to instrumental drifts (Patanchon et al. 2008).

The bolometers in each array are corrected for relative gains, or flat-fielded, so that multi-bolometer maps can be generated. The flat-field corrections are determined by making individual maps for each bolometer from a single point-source calibrator, in this case, VY CMa (see Section 5.1). The bolometers are also corrected for responsivity variations over time by using the signals from a calibration lamp in the optics box, which was pulsed at every 15 minutes throughout the observations. The resulting signal is used to correct any time-varying changes in responsivity per bolometer. Both the time-varying changes and the variations of beams across each array are small, and the amount of variation is comparable to those detailed in Section 2 of T08 for BLAST05.

To calculate the flux density from a point source, we adopt a matched-filtering technique similar to that used to extract point sources from several recent extragalactic submillimeter surveys (e.g., Coppin et al. 2006; Scott et al. 2006) and detailed in Section 2 of T08. The beam profile on the sky, or pointspread function (PSF), used for calibration and flux extraction is generated by stacking and averaging several observations of VY CMa after removing the background. The PSF is normalized as in Equation (1) of T08, and if maps are further filtered, the PSF used for analysis of that map must also be filtered after normalization. See Section 5.1 for discussion of the backgroundsubtraction technique.
Because the BLAST filters have wide (30\%) spectral bands (Pascale et al. 2008, Section 2.5 and Figure 2), the derived flux densities depend on the shape of the source spectrum within the band, and thus on the temperature of the source. A correction must be made to calculate monochromatic flux densities from the maps. See Section 5.1 of T08 for details of this color correction.

\section{BLAST06 WARM OPTICS PERFORMANCE}

The BLAST06 warm optics (primary and secondary mirrors) performed within their specifications. The BLAST06 optical performance was very much improved over that of BLAST05 through the use of a new aluminum primary mirror as well as an in-flight focusing system (Pascale et al. 2008, Section 2.4). PSFs for each of the BLAST bands are shown in Figure 1.

With the pre-flight predicted noise-equivalent flux density (NEFD) of $220 \mathrm{mJy} \mathrm{s}^{1 / 2}$, we would expect $1 \sigma$ surface brightness fluctuations at the nominal resolutions of $\sim \mathrm{NEFD} / \Omega$, or $11,4.7$, and $2.6 \mathrm{MJy} \mathrm{sr}^{-1} \mathrm{~s}^{1 / 2}$ at 250,350 , and $500 \mu \mathrm{m}$, respectively, where $\Omega$ is the area of the beam $\left(\mathrm{FWHM}^{2}\right)$. The measured sensitivities in each band were 8.8, 4.8, and 2.7 $\mathrm{MJy} \mathrm{sr}^{-1} \mathrm{~s}^{1 / 2}$, respectively, which demonstrates that both the detectors and optical efficiencies largely achieved our design goals. The only exception is the $250 \mu \mathrm{m}$ beam, which is slightly larger than the diffraction limit, and has significant sidelobes. For each PSF, $76 \%, 92 \%$, and $>95 \%$ of the power is located within a Gaussian fit to the PSF, which have FWHM of $36^{\prime \prime}, 42^{\prime \prime}$, and $60^{\prime \prime}$ at 250 , 350 , and $500 \mu \mathrm{m}$, respectively. See Table 1 . See Section 3 and Figure 4 of Pascale et al. (2008) for further details on the noise and sensitivity of the BLAST detectors, and see Chattopadhyay et al. (2003) and Rownd et al. (2003) for a detailed description of the detectors.

\section{BLAST06 POINTING PERFORMANCE}

Pointing is measured in-flight to an accuracy of $\sim 30^{\prime \prime} \mathrm{rms}$ using a combination of fine and coarse sensors, including fiber-optic gyroscopes, optical star cameras, a differential GPS, magnetometer, and Sun sensor (Pascale et al. 2008, Section 7). Post-flight pointing reconstruction uses only the gyroscopes and day-time star cameras. The algorithm is based on a similar multiplicative extended Kalman filter technique used by WMAP (Pittelkau 2001; Markley 2003), modified to allow the evaluation of the alignment parameters of the star cameras and gyroscopes 
Table 1

Calibration and Performance Parameters for BLAST06

\begin{tabular}{|c|c|c|c|c|c|c|}
\hline \multirow{2}{*}{$\begin{array}{l}\text { Band } \\
(\mu \mathrm{m})\end{array}$} & \multirow{2}{*}{$\begin{array}{l}\text { Calib. Coeff. } \\
\left(\times 10^{12} \mathrm{Jy} \mathrm{V}^{-1}\right)\end{array}$} & \multirow{2}{*}{$\begin{array}{c}\text { Uncertainty }^{\mathrm{a}} \\
(\%)\end{array}$} & \multicolumn{3}{|c|}{ Pearson Correlation Matrix ${ }^{\mathrm{b}}$} & \multirow{2}{*}{$\begin{array}{c}\text { PSF FWHM } \\
(\operatorname{arcsec})\end{array}$} \\
\hline & & & $250 \mu \mathrm{m}$ & $350 \mu \mathrm{m}$ & $500 \mu \mathrm{m}$ & \\
\hline 250 & 2.73 & 9.5 & 1 & 0.83 & 0.80 & 36 \\
\hline 350 & 2.86 & 8.7 & & 1 & 0.83 & 42 \\
\hline 500 & 1.16 & 9.2 & & & 1 & 60 \\
\hline
\end{tabular}

Notes. Calibration coefficients, calibration uncertainties, and Pearson correlation matrix, showing the relationship between errors in different bands, for BLAST06. Note that high correlation means that the measurements involving the ratio of BLAST brightnesses, such as spectral indices and temperature, can be reported with much higher accuracy. Also included are the measured FWHM of each beam determined by fitting a Gaussian; $1 \sigma$ errors on the FWHM are of order $1^{\prime \prime}$.

${ }^{a}$ These values include the 5\% uncertainty estimated for the bandpass measurements. Without them, they are $8.1 \%, 7.1 \%$, and $7.8 \%$ at 250,350 , and $500 \mu \mathrm{m}$, respectively.

b These values include the $5 \%$ uncertainty correlated at $50 \%$ between bands for the bandpass measurements. Without them, they are 0.977 between 250 and $350 \mu \mathrm{m}, 0.917$ between 250 and $500 \mu \mathrm{m}$, and 0.981 between 350 and $500 \mu \mathrm{m}$.

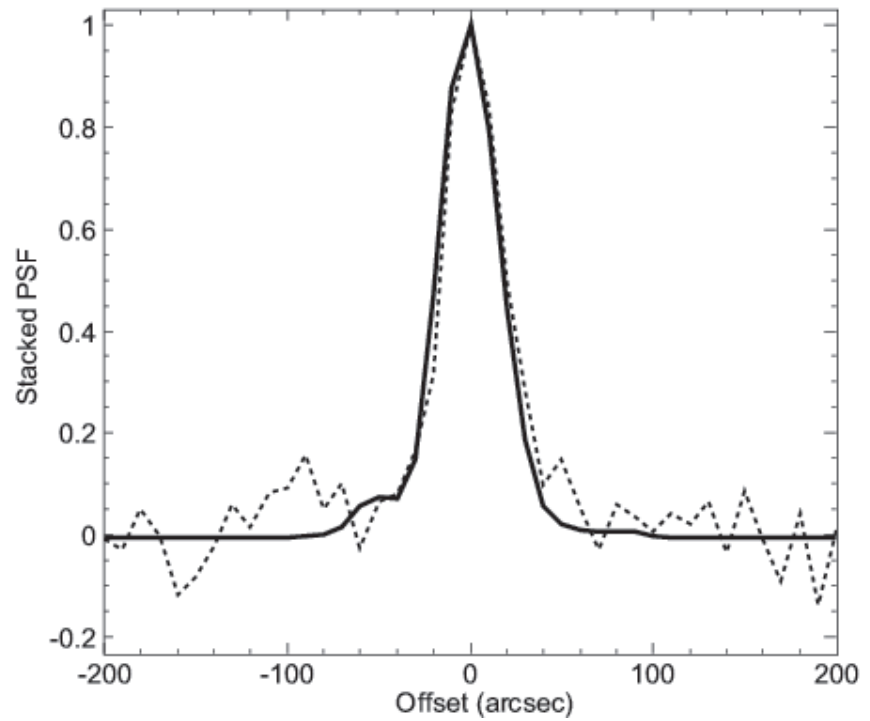

Figure 2. Cut through the peak-normalized stacked BLAST $250 \mu \mathrm{m}$ map at the positions of VLA 1.4 GHz radio sources (dashed line) and through the $250 \mu \mathrm{m}$ PSF (solid line). We see that the stack is very well described by the PSF, in both position and width. We conclude that our absolute pointing is good to $2^{\prime \prime}$ and that random pointing errors are $<5^{\prime \prime} \mathrm{rms}$.

(Pascale et al. 2008, Section 11). The offset between the star cameras and the submillimeter telescope was measured by repeated observations of pointing calibrators throughout the flight. We find that the relative pointing between the star cameras and submillimeter telescope varies as a function of telescope elevation. We apply an elevation-dependent correction to pitch and yaw with approximate peak-to-peak amplitudes of $260^{\prime \prime}$ and $\sim 36^{\prime \prime}$, respectively, over the full $25^{\circ}-60^{\circ}$ elevation range of the telescope.

Post-flight pointing accuracy is verified by a stacking analysis on one of the extragalactic maps. Using the deep radio Extended Chandra Deep Field-South Very Large Array survey at $1.4 \mathrm{GHz}$ (Miller et al. 2008) we stack patches of the BLAST maps centered at the radio source coordinates, summing the flux density pixel by pixel (see Figure 2). We find that the peak in the stacked map is located within $2^{\prime \prime}$ from the nominal position of the catalog, more than 15 times smaller than the beam size. Moreover, assuming random Gaussian pointing errors, we superimpose the synthetic scaled PSF on the stacked map and convolve it with a Gaussian profile, modeling the broadening of the PSF due to pointing jitter. By varying the jitter width, we compute the $\chi^{2}$ of the convolved PSF over the stacked data. In this way, we estimate the upper limit in potential random pointing errors to be $<5^{\prime \prime}$.

\section{ASTRONOMICAL FLUX CALIBRATION}

The primary scientific goals of the BLAST experiment demand an absolute flux calibration accuracy of $\sim 10 \%$ in all three BLAST passbands. Achieving this was complicated by the variability in which part of the sky was visible to BLAST due to the unstable projected latitude of the telescope gondola during the flight, the restrictions on visibility due to the Sun and Moon avoidance criteria, the orientation of Sun shields and other baffling, and the elevation range $\left(25^{\circ}-60^{\circ}\right)$ of the gondola's inner-frame (Pascale et al. 2008). Consequently, BLAST had only limited access to the calibration sources commonly used at submillimeter and far-infrared (FIR) wavelengths.

Since the ecliptic plane was not visible during the BLAST06 flight, no absolute flux calibration could be determined from observations of Uranus or Mars, for which model SEDs are known to have systematic uncertainties $<5 \%$ at submillimeter wavelengths (Griffin \& Orton 1993; Wright 2007). The preflight strategy for achieving a 10\% calibration accuracy required the identification of alternate Galactic and extragalactic sources that could act as primary and secondary calibrators. The requirements included (1) availability throughout the flight; (2) considered, in some cases, as secondary calibrators for ground-based submillimeter telescopes and FIR satellites; (3) well-constrained SEDs in the FIR to millimeter-wavelength regime, enabling accurate interpolation of the band-averaged flux densities at BLAST wavelengths; and (4) bright ( $\gg 1 \mathrm{Jy}$ at $500 \mu \mathrm{m}$ ) and compact sources (with respect to the BLAST beam size, i.e., $<20^{\prime \prime}$ ) that reside in regions with minimal spatial structure in the Galactic foregrounds or backgrounds, allowing accurate subtraction of any extended emission. Given these criteria, we scheduled regular observations throughout the flight of bright embedded protostellar-sources and compact $\mathrm{H}$ II regions, as well as the star VY CMa. For calibration purposes, we assume that the SEDs are smooth and neglect any contribution from molecular emission lines (see Section 4.1 of T08).

\subsection{VY CMa-The Primary Calibrator for BLAST06}

We chose VY CMa, a red hypergiant star, as our primary calibrator. It was the most isolated, point-like bright object available during the flight. It is located in the Galactic Plane 
Table 2

Submillimeter flux densities for VY CMa

\begin{tabular}{lcccc}
\hline \hline $\begin{array}{l}\text { Wavelength } \\
(\mu \mathrm{m})\end{array}$ & $\begin{array}{c}\text { Flux Density } \\
(\mathrm{Jy})\end{array}$ & $\begin{array}{c}\text { Error } \\
(\mathrm{Jy})\end{array}$ & Instrument & Reference \\
\hline 12 & 9919 & 992 & IRAS & Helou \& Walker (1988) \\
25 & 6651 & 665 & $I R A S$ & Helou \& Walker (1988) \\
60 & 1453 & 145 & $I R A S$ & Helou \& Walker (1988) \\
100 & 331 & 33 & $I R A S$ & Helou \& Walker (1988) \\
350 & 15.1 & 1.5 & SHARC II & D. Dowell (2007, private communication) \\
450 & 9.7 & 4.9 & UKT 14 & Sandell (1994) \\
800 & 2.81 & 0.4 & UKT 14 & Sandell (1994) \\
850 & 2.13 & 0.3 & SCUBA & T. Jenness (2007, private communication) \\
1100 & 0.75 & 0.08 & BOLOCAM & J. Aguirre (2007, private communication) \\
$250^{\mathrm{a}}$ & 37.4 & 3.7 & BLAST & This paper \\
$350^{\mathrm{a}}$ & 15.0 & 1.7 & BLAST & This paper \\
$500^{\mathrm{a}}$ & 6.66 & 0.9 & BLAST & This paper \\
\hline
\end{tabular}

Notes. Flux densities used to generate the VY CMa SED shown in Figure 4 as discussed in the text. Flux densities for BLAST and from IRAS have been color corrected. The error column shows photometric and calibration uncertainties added in quadrature for each non-BLAST measurement; only calibration uncertainty is shown for BLAST.

${ }^{a}$ Extracted from the fit and used to calibrate BLAST06.

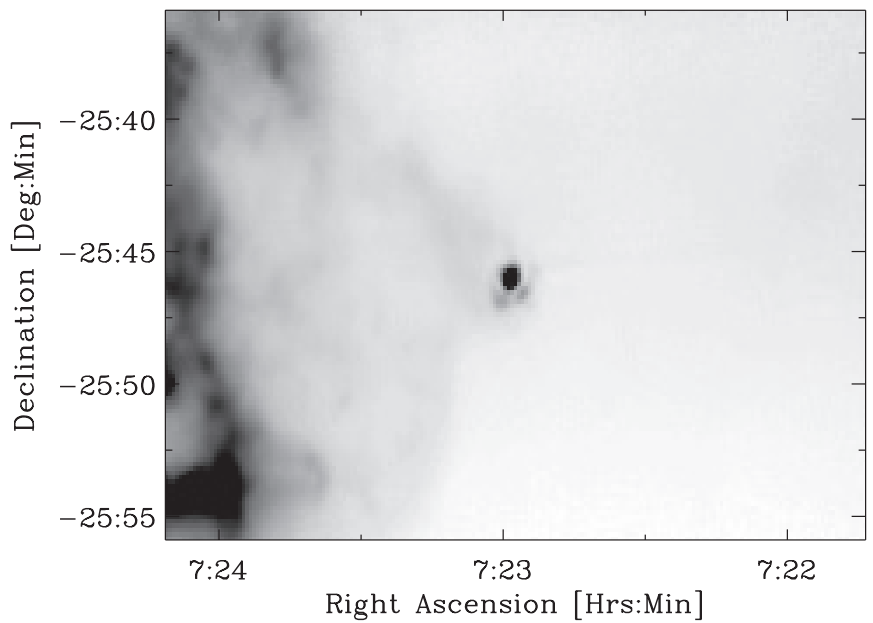

Figure 3. BLAST map of VY CMa at $250 \mu \mathrm{m}$. This map is in sky coordinates and does not have the background removed.

at R.A. $7^{\mathrm{h}} 22^{\mathrm{m}} 58^{\mathrm{s}} 3$, decl. $-25^{\circ} 46^{\prime} 3^{\prime \prime} .2(\mathrm{~J} 2000)$ and is one of the most intrinsically luminous stars known (Humphreys et al. 2007; Choi et al. 2008). Unfortunately, it is not entirely pointlike due to the Galactic cirrus, which significantly complicates calibration. Figure 3 shows the BLAST VY CMa map. An accurate PSF is required to use the calibration method as outlined above and in T08. The procedure used to remove the background around VY CMa is discussed below, in Section 5.2.

Data are collated to generate the FIR SED used to calibrate BLAST06 in a manner similar to Arp 220 from BLAST05 (T08, Section 4.1; see Table 2 and the SED in Figure 4). These data consist of measurements from IRAS at 12, 25, 60, and $100 \mu \mathrm{m}$ (Helou \& Walker 1988), from SCUBA on the JCMT at $850 \mu \mathrm{m}$ (T. Jenness 2007, private communication), from SHARC-II on the CSO at $350 \mu \mathrm{m}$ (D. Dowell 2007, private communication), from Bolocam on the CSO at $1.1 \mathrm{~mm}(\mathrm{~J}$. Aguirre 2007, private communication), and from UKT14 on the JCMT at 450 and $800 \mu \mathrm{m}$ (Sandell 1994). Careful attention has been placed on calculating the full correlation matrix of the errors, as discussed in Section 4.1 of T08. Briefly, measurements from the same instrument are taken to be $100 \%$ correlated (in

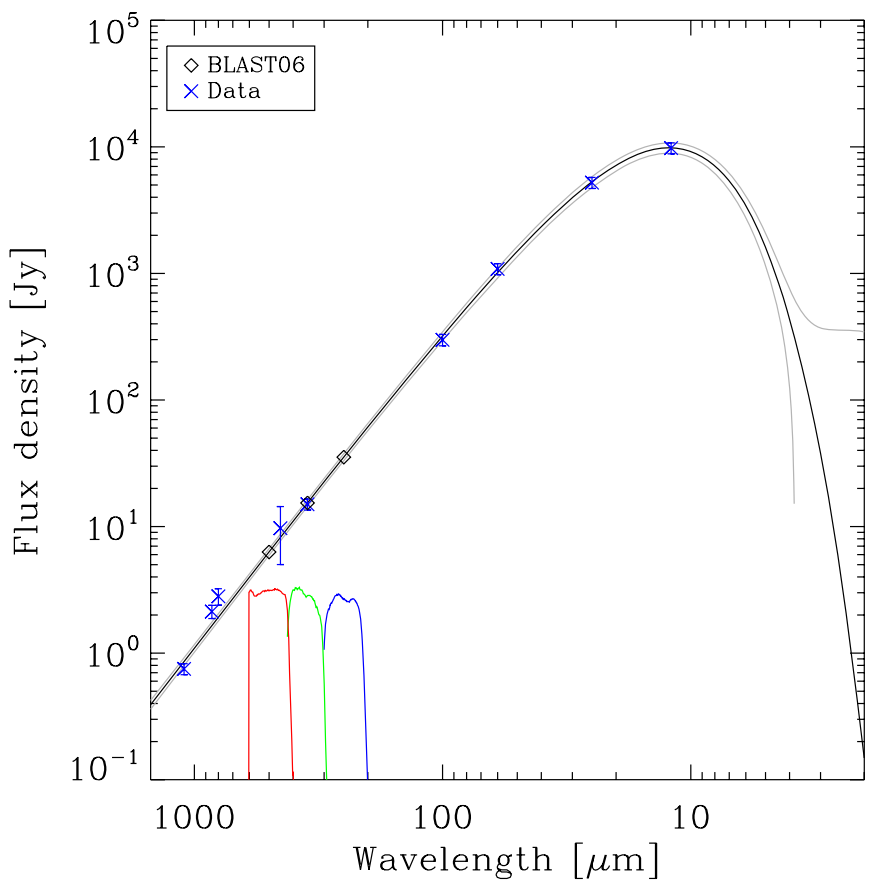

Figure 4. SED of VY CMa, the absolute flux calibrator for BLAST06. The best-fit model (heavy solid line) is constrained using the published data (blue crosses, discussed in Section 5.1, listed in Table 2), excluding BLAST06 measurements. The gray lines show the $68 \%$ confidence interval, estimated from 100 Monte Carlo simulations, about the best-fit model. Black diamonds indicate model predictions for BLAST06 at 250, 350, and $500 \mu \mathrm{m}$. The $1 \sigma$ uncertainties associated with these predictions are $8.1 \%, 7.1 \%$, and $7.8 \%$ in each band, respectively, which are highly correlated. For reference, the three BLAST passbands are shown (normalized to an arbitrary amplitude on the plot). (A color version of this figure is available in the online journal.)

addition to statistically independent photometric uncertainties), and all measurements are assumed to have an additional fully correlated error of $5 \%$, since the bulk of these instruments were all calibrated to the same Uranus SED (Griffin \& Orton 1993).

We find a best-fit model with parameters $T=346 \pm 19 \mathrm{~K}$, $\beta=0.545 \pm 0.046$, and $S_{\mathrm{FIR}}=(3.42 \pm 0.38) \times 10^{17} \mathrm{~W} \mathrm{~m}^{-2}$ (see Figure 4). This results in BLAST calibration uncertainties of $8.1 \%, 7.1 \%$, and $7.8 \%$ at 250,350 , and $500 \mu \mathrm{m}$, 
respectively. These are highly correlated, with Pearson correlation coefficients of $\rho_{250-350}=0.977, \rho_{250-500}=0.917$, and $\rho_{350-500}=0.981$. See Table 1 . The large degree of correlation in these values shows that almost all of the uncertainty in the SED is in the absolute value. The relative calibration uncertainty between different BLAST bands (i.e., color uncertainty), on the other hand, is actually very small, and exceeds the requirements for the experiment. In fact, care must be taken when fitting simple model SEDs to high-S/N measurements where the calibration uncertainties dominate. In such cases, the BLAST data may place such stringent constraints on SED shapes (e.g., slope and curvature through the BLAST bands) that single-temperature thermal SEDs are not consistent with the observations. Section 4.5 of Wiebe et al. (2009) discusses how to incorporate the bandpass uncertainties for high-S/N sources.

We include an additional calibration uncertainty related to our measurement of the BLAST spectral bandpasses. Since we have used our measured bandpasses to calculate the incident power on our detectors given the SED of our primary calibrator, any sources with different SEDs could have uncertainties in their reported band-averaged flux densities arising from uncertainties in the BLAST bandpass measurements (see Equation (5) of T08). The dominant source of uncertainty in this measurement is our knowledge of the location of the band edges (see Figure 2 of Pascale et al. 2008), which we believe to be no greater than about 5\%. Assuming this maximum uncertainty in the measurement, we have calculated the effect it would have on sources with different SEDs, ranging from "hot" sources ( $T>40 \mathrm{~K})$, to "cool" sources $(T \approx 15 \mathrm{~K})$. Since all of the BLAST filters sample the Rayleigh-Jeans regime of VY CMa, the SEDs of "hot" sources resemble the primary calibrator, and this uncertainty has no effect. However, for "cool" sources, the SED turns over, particularly in the $250 \mu \mathrm{m}$ band. We, therefore, include a $5 \%$ uncertainty due to the uncertainty in the knowledge of the bandpasses. Since the bandpasses were measured simultaneously with the same Fourier transform spectrometer, there may be significant band-band correlations in the bandpass measurements. We assume correlation coefficients of $50 \%$ between all bands.

\subsection{VY CMa Background Subtraction}

A complication with the use of VYCMa as a calibrator is that it is not entirely point-like due to Galactic cirrus. We attempt to use the cross-linking (limited to an angle between scans of only $9^{\circ}$ due to the extreme southerly flight of BLAST06) of the region in order to separate the beam shape from the diffuse background emission. The former is coherent in telescope coordinates, while the latter is coherent in sky coordinates. By iterating between the two coordinate systems, it is possible to better separate the two components, since features in the sky are smoothed out to lower significance levels in telescope coordinates and features in the PSF are smoothed out to lower significance in sky coordinates.

A zeroth-order background subtracted map is generated by extrapolating the surrounding emission into the region of the point source. A low-order two-dimensional polynomial is fitted to the map with the source masked out.

We start with the true data stream, $d$, and an initial estimate of the PSF, $P_{0}$, taken from the zeroth-order background subtracted maps. From there, we scan the estimate of the point source (PSF), $P_{i}$, using a sky simulator (an inverse map-maker which takes maps and the BLAST pointing solution and generates raw time streams). The iteration index is denoted by $i$. The PSF maps are in telescope coordinates, so scanning must also be done in telescope coordinates. The result is a simulated PSF-only data stream, $p_{i}^{*}$. We then subtract $p_{i}^{*}$ from the true data stream, $d$, producing the background-only data stream, $b_{i}=d-p_{i}^{*}$. Then we create a point-source subtracted background map, $B_{i}$, from the background-only data stream. This map is in sky coordinates. The central region of the background-only map, $B_{i}$, can be optionally smoothed, but this is only done in the first iteration. Next, the estimate of background, $B_{i}$, is scanned with the sky simulator, producing the simulated data stream, $b_{i}^{*}$. Then, the background-only data stream is subtracted from the true data stream, producing the PSF-only data stream, $p_{i}=d-b_{i}^{*}$. Finally, a new estimate of the PSF, $P_{i+1}$, is made using the simulated data stream $p_{i}$.

We performed 10 iterations of this algorithm in all three bands. We examined the rms in the beam difference maps between iterations, finding that it was less than $1 \%$ of the rms in the total beam map for the final iteration. In addition, the ratio of these quantities was shown to drop monotonically, with most of the decrease from $\sim 20 \%$ to $1 \%$ occurring in the first 2-5 iterations, after which it flattened out, demonstrating that the answer had converged.

We note that the most complicated beam pattern is at $250 \mu \mathrm{m}$. However, the final answer is least sensitive to the details of the background subtraction at this wavelength because the point-source contrast increases. Since the beam is smaller, the brightness in a beam of the diffuse background is reduced. Furthermore, the diffuse dust in the interstellar medium has a temperature of about $20 \mathrm{~K}$ (e.g., Schlegel et al. 1998), whereas VY CMa has a much warmer effective SED in the BLAST bands of $200 \mathrm{~K}$, and is therefore much brighter at $250 \mu \mathrm{m}$.

\section{CONCLUSIONS}

For the BLAST06 flight, improved optics over the BLAST05 flight yielded improved point-source sensitivity. BLAST06 calibration uncertainties are approximately $10 \%$, as expected. Since we calibrate all BLAST bands to one object (VY CMa), the calibration uncertainties are highly correlated; this high correlation should be taken into account when using BLAST photometry measurements to constrain SEDs.

Our absolute post-flight reconstructed pointing has been shown to be good to $2^{\prime \prime}$ with random pointing errors $<5^{\prime \prime}$. Together with the fact that BLAST06 uncertainties are typically much smaller than those of other experimental measurements in the 250-500 $\mu \mathrm{m}$ waveband, this should make BLAST data useful as a cross-correlation check for future submillimeter experiments. Future observations of VY CMa with the SPIRE instrument on Herschel (Griffin et al. 2004) would provide a useful cross-check of the calibration here, and the higher resolution would improve the background subtraction.

We acknowledge the support of NASA through grant Nos. NAG5-12785, NAG5-13301, and NNGO-6GI11G, the NSF Office of Polar Programs, the Canadian Space Agency, the Natural Sciences and Engineering Research Council (NSERC) of Canada, and the UK Science and Technology Facilities Council (STFC). We thank D. Dowell, T. Jenness, and J. Aguirre for their data. This research has been enabled by the use of WestGrid computing resources. This research also made use of the SIMBAD database, operated at the CDS, France, and the NASA/IPAC Extragalactic Database (NED), operated by the Jet Propulsion Laboratory, under contract with NASA. 


\section{REFERENCES}

Chattopadhyay, G., Glenn, J., Bock, J. J., Rownd, B. K., Caldwell, M., \& Griffin, M. J. 2003, IEEE Trans. Micro. Theory and Tech., 51, 2139

Choi, Y. K., et al. 2008, PASJ, 60, 1007

Coppin, K., et al. 2006, MNRAS, 372, 1621

Devlin, M. J., et al. 2009, Nature, 458, 737

Griffin, M. J., \& Orton, G. S. 1993, Icarus, 105, 537

Griffin, M. J., Swinyard, B. M., \& Vigroux, L. 2004, Proc. SPIE, 5487, 413

Guthnick, P., \& Schneller, H. 1939, Astron. Nachr., 268, 165

Helou, G., \& Walker, D. W. (ed.) 1988, Infrared Astronomical Satellite (IRAS) Catalogs and Atlases (Washington, DC: NASA)

Hoffmeister, C. 1931, Astron. Nachr., 242, 129

Hughes, D. H., et al. 2002, MNRAS, 335, 871

Humphreys, R. M., Helton, L. A., \& Jones, T. J. 2007, AJ, 133, 2716
Markley, F. L. 2003, J. Guidance, Control, Dyn., 26, 311

Miller, N. A., Fomalont, E. B., Kellermann, K. I., Mainieri, V., Norman, C., Padovani, P., Rosati, P., \& Tozzi, P. 2008, ApJS, 179, 114

Netterfield, C. B., et al. 2009, ApJ, 707, 1824

Pascale, E., et al. 2008, ApJ, 681, 400

Patanchon, G., et al. 2008, ApJ, 681, 708

Pittelkau, M. E. 2001, J. Guidance Control Dyn., 24, 1187

Rownd, B., Bock, J. J., Chattopadhyay, G., Glenn, J., \& Griffin, M. J. 2003, Proc. SPIE, 4855,510

Sandell, G. 1994, MNRAS, 271, 75

Schlegel, D. J., Finkbeiner, D. P., \& Davis, M. 1998, ApJ, 500, 525

Scott, D., et al. 2006, BAAS, 38, 1072

Truch, M. D. P., et al. 2008, ApJ, 681, 415

Wiebe, D. V. et al. 2009, ApJ, 707, 1809

Wright, E. L. 2007, arXiv:astro-ph/0703640 